\title{
Overexpression of DJ-1 alleviates autosomal dominant polycystic kidney disease by regulating cell proliferation, apoptosis, and mitochondrial metabolism in vitro and in vivo
}

\author{
Zhongxin Li ${ }^{1,2}$, Jingjing Zhou ${ }^{2}$, Yan $\mathrm{Li}^{2}$, Fan Yang ${ }^{2}$, Xiaoying Lian', Wenhu Liu ${ }^{1}$ \\ ${ }^{1}$ Department of Nephrology, Beijing Friendship Hospital, Capital Medical University, Beijing, China; ${ }^{2}$ Department of Nephrology, Beijing Luhe \\ Hospital, Capital Medical University, Beijing, China \\ Contributions: (I) Conception and design: Z Li, W Liu; (II) Administrative support: W Liu; (III) Provision of study materials or patients: Z Li, J Zhou; \\ (IV) Collection and assembly of data: J Zhou, Y Li, X Lian; (V) Data analysis and interpretation: Z Li, F Yang; (VI) Manuscript writing: All authors; \\ (VII) Final approval of manuscript: All authors. \\ Correspondence to: Wenhu Liu. Department of Nephrology, Beijing Friendship Hospital, Capital Medical University, No. 95 Yong An Road, Xi Cheng \\ District, Beijing, China. Email: liuwenhu2013@163.com.
}

\begin{abstract}
Background: $D f-1$ is critical for the mitochondrial function associated with autosomal dominant polycystic kidney disease (ADPKD). We aimed to investigate Df-1's function in the pathogenesis of ADPKD.

Methods: Df-1 was knocked-down in IMCD3 cells to evaluate the effects of $D F-1$ on cell phenotype and mitochondrial function in vitro. Furthermore, we generated three groups of mice with different expression levels of DJ-1 within an established ADPKD model: ADPKD, ADPKD ${ }^{\mathrm{pcDNA}}$, and ADPKD ${ }^{\mathrm{pcDNA}-\mathrm{DJ}-1}$.

Results: Df-1 knock-down significantly increased oxidative stress as well as the proliferation and apoptosis rate of IMCD3 cells, along with Bcl-2 down-regulation and the up-regulation of Ki67, PCNA, Bax, cleaved caspase-3, and cleaved caspase-9. Df-1 knock-down suppressed the cellular respiration, $\mathrm{Ca}^{2+}$ absorption, and mitochondrial complex I activity in mitochondria. In vivo, we verified that DJ-1 was down-regulated in ADPKD models, and its overexpression attenuated the renal dysfunction in ADPKD models. The transgenic mice had a significantly smaller renal cyst and less interstitial fibrosis than control, accompanied by $\alpha$-SMA, fibronectin, and TGF- $\beta 1$ up-regulation. Moreover, in vivo results confirmed DJ-1 overexpression inhibited the proliferation and apoptosis of tubular epithelial cells along with down-regulation of Ki67, PCNA, p53, intracellular Cyt c, cleaved caspase-3, and cleaved caspase-9 and the up-regulation of Bcl-2.

Conclusions: DJ-1 was down-regulated in ADPKD models, and its overexpression may attenuate the renal dysfunction and pathological damage by regulating the proliferation, apoptosis, oxidative stress and mitochondrial metabolism, which may be mediated by the p53 signaling pathway.
\end{abstract}

Keywords: Autosomal dominant polycystic kidney disease (ADPKD); Df-1; mitochondrial metabolism; mitochondrial dysfunction

Submitted Jul 22, 2020. Accepted for publication Sep 04, 2020.

doi: 10.21037/atm-20-5761

View this article at: http://dx.doi.org/10.21037/atm-20-5761

\section{Introduction}

Autosomal dominant polycystic kidney disease (ADPKD) is the most prevalent, potentially lethal, monogenic, inherited kidney disease, which accounts for approximately $10 \%$ of all patients on renal replacement therapy worldwide $(1,2)$. Abnormalities characterize ADPKD in epithelial cell growth, leading to the formation of multiple renal cysts and progressive renal failure (3). The remarkable advances in the diagnosis, prognosis, and treatment of ADPKD have been achieved over the past ten decades. However, this disorder remains to be identified as untreatable, relentlessly progressing towards end-stage renal disease (4). 
Unfortunately, its pathogenesis is poorly understood. Herein, the elucidation of underlying cellular and genetic mechanisms of ADPKD is urgently needed and would contribute to providing a foundation for the development of potentially effective treatments and clinical trials.

Recently, emerging evidence demonstrated that the reduction of intracellular calcium $\left(\mathrm{Ca}^{2+}\right)$ in ADPKD could affect mitochondrial function and ATP production with a consequence of glucose metabolism (5). Mitochondrial dysfunction exists from an early phase of ADPKD, suggesting a relationship between mitochondria function and ADPKD $(6,7)$. More recently, as the study reported by Ishimoto et al., mitochondrial dysfunction facilitates the cyst formation in $\operatorname{ADPKD}(8,9)$, further indicating the vital function of mitochondria in cyst formation. It is widerecognized that there exists a structural and functional link between the mitochondrial network and the endoplasmic reticulum (ER) (10,11). A vital aspect of this relationship is the modulation of $\mathrm{Ca}^{2+}$ signaling during cell activation, which could accordingly affect various biological and physiological processes $(12,13)$. Notably, it is reported that the defective mitochondrial $\mathrm{Ca}^{2+}$ transfer may contribute to the aberrant cell proliferation of kidney cysts in ADPKD (14). Thus, the regulation of mitochondria function may supply a novel perspective on the molecular mechanisms of ADPKD.

DJ-1 belongs to the ThiJ/PfpI protein superfamily and is a cytoprotective protein implicated in many cellular processes, which plays a pivotal role in transcriptional regulation and anti-oxidative stress (15). Previously, many studies indicated that mutations in the $D f-1$ gene result in recessive parkinsonism (16). Interestingly, $D \mathcal{F}-1$ is proved to be critical for mitochondrial function (17), which also has a close connection with ADPKD. The knock-down of Df-1 impairs the mitochondrial function of astrocyte (18) and could maintain the mitochondrial function in an oxidative environment (19). Moreover, the protective effects of $D f-1$ on mitochondrial function and disease progression have been elaborated in various diseases' biology beyond Parkinson's disease, including ischemia-reperfusioninduced heart failure (20) and cancer biology (21). Although numerous studies revealed the protective effects of DJ-1 on mitochondrial function in a variety of diseases, so far, no studies have been conducted to elucidate the role of DJ-1 in molecular mechanisms of ADPKD. Given that relationship between mitochondria function and $\operatorname{ADPKD}(6,7)$, we hypothesized that $D \mathcal{f}-1$ might support the mitochondrial function, thus alleviating the progress of ADPKD. To the best of our knowledge, this is the first study to investigate the effects of $D f-1$ on the disease progression of ADPKD.

Herein, we creatively attempt to verify the effects of Df-1 on cell proliferation, apoptosis, and mitochondrial metabolism in mouse inner medullary collecting duct lines. Meanwhile, the protective effects of $D \mathcal{F}-1$ on ADPKD were further investigated tentatively, expecting to supply a novel therapeutic target for ADPKD therapy. We present the following article in accordance with the ARRIVE reporting checklist (available at http://dx.doi.org/10.21037/atm-205761).

\section{Methods}

\section{In vivo experimental design}

\section{Animals}

All the mice models used in this study were from a C57BL/6J inbred background. Pkd1fl/fl mice were bred with pkhd-cre (+) mice to generate APPKD mouse models. The $D \mathcal{f}-1$ sequence was cloned into a pcDNA4-vector to obtain the transgenic mice ADPKD model overexpressing DJ-1 (each group, $\mathrm{n}=10$ ). The transgenic ADPKDpcDNADJ-1 mouse was engineered by the Model Animal Research Center of Nanjing University (Nanjing, Jiangsu, China). All mice were provided with a standard diet and housed in a 12-hour light/dark cycle. All animal procedures were performed following the NIH Guide for the Care and Use of Laboratory Animals and approved by the Ethics Review Committee of Beijing Friendship Hospital, Capital Medical University \{approval number: SYXK[Jing]2017-0019\}.

\section{Renal function tests}

Before sacrificing the mice, the animals were weighed, and blood was collected to measure creatinine, blood urea nitrogen (BUN), uric acid, and proteinuria levels. Serum creatinine was measured using capillary electrophoresis. The levels of BUN, uric acid, and proteinuria were detected using the Cobas C311 automated biochemistry analyzer (Roche Diagnostics, Germany).

\section{Tissue harvesting and histopathologic analysis}

Mice were sacrificed, and kidney tissue was obtained. The kidney tissues were at once fixed in $10 \%$ buffered formalin for 24 hours and embedded in paraffin. For the histopathological analysis, tissues were sliced into $5 \mu \mathrm{m}$ thick sections. Then, hematoxylin \& eosin (H\&E) staining and Masson staining were respectively performed according to the standard protocol. The sections were visualized 
and analyzed under a microscope (Leica, Japan). The percentages of the stained area with Masson's ponceau acid fuchsin solution were calculated using Image J software.

Terminal-deoxynucleotidyl Transferase Mediated Nick End Labeling (TUNEL) staining

Apoptosis of Bowman's capsule epithelial cells (BCECs) was detected by in situ TUNEL assay using a commercially available kit (Roche Diagnostics, Germany). Briefly, after paraffin sections were deparaffinized and rehydrated, endogenous peroxidase activity was quenched in 3\% hydrogen peroxide for $20 \mathrm{~min}$. Sections were treated with proteinase $\mathrm{K}$ and were labeled with $\mathrm{TdT}$ and biotinylated dUTP. Washed sections were incubated with peroxidaselabeled streptavidin for $30 \mathrm{~min}$ and then stained with diaminobenzidine (DAB), followed by counterstaining with hematoxylin. Finally, the TUNEL-positive cells were counted using ImageJ software.

\section{Immunohistochemistry (IHC)}

IHC staining was performed using a standard streptavidinperoxidase method. After deparaffinization and unmasking epitopes, kidney sections were incubated with primary antibody against cleaved caspase 3 (1:500, ab2302, Abcam, Cambridge, MA, USA) at $4{ }^{\circ} \mathrm{C}$ overnight, followed by incubation with the secondary antibody for $20 \mathrm{~min}$ at room temperature. Then, kidney sections were incubated with horseradish peroxidase (HRP)-conjugated streptavidin peroxidase (1:40,000, ab7403; Abcam) for $30 \mathrm{~min}$. DAB was used as the chromogen to visualize the results. Finally, kidney sections were counterstained with hematoxylin.

\section{In vitro experimental design}

\section{Cell line and culture}

Mouse inner medullary collecting duct cell lines (IMCD3) were obtained from the American Type Culture Collection (ATCC, USA) and grown in DMEM low glucose medium supplemented with $10 \%$ fetal bovine serum (FBS, Gibco, USA) at $37{ }^{\circ} \mathrm{C}$ in a humidified incubator with a $5 \% \mathrm{CO}_{2}$ atmosphere.

Cell transfection, proliferation, apoptosis and oxidative stress assays

IMCD 3 cells were grown in a six-well plate $\left(2 \times 10^{5}\right.$ cells per well) with the Dulbecco's Modified Eagle Medium (DMEM). At the 80-90\% confluence, cells were transfected with plasmid DJ-1-shRNA or a control shRNA using
Lipofectamine 2000 Transfection Kit (Invitrogen, USA) following the manufacturer's instruction. Stable transfectants (clones) were selected, as described elsewhere (22). Two days after transfection, the cells were transferred to a 96-well plate and incubated in a $\mathrm{CO}_{2}$ incubator at $37^{\circ} \mathrm{C}$ for 24 hours. For the proliferation assay, cell counting kit-8 (CCK-8) solution $(10 \mu \mathrm{L})$ was added to each well and incubated for another two hours. Finally, the absorbance was measured by at $450 \mathrm{~nm}$ using a $\mu$ Quant MQX200 microplate reader (Bio-Tek Instruments, USA). For apoptosis assay, the cell apoptosis rate was detected using Annexin $\mathrm{V}$-fluorescein isothiocyanate (FITC) apoptosis kit (BD Biosciences, San Jose, CA, USA) according to the standard protocol of flow cytometry assay. Briefly, two days after transfection, the prepared cells were double-stained with $5 \mu \mathrm{L}$ Annexin $\mathrm{V}$-FITC and $5 \mu \mathrm{L}$ propidium iodide for $15 \mathrm{~min}$ at room temperature. The apoptosis rate was at once measured in a FACSAria $^{\mathrm{TM}}$ Fusion flow cytometer and analyzed using the RNA CellQuest software (BD Biosciences, San Jose, CA, USA). Malondialdehyde (MDA) Assay Kit and Superoxide Dismutase (SOD) Activity Assay Kit (Abcam, USA) were used to measure the levels of MDA and SOD, following manufacturer's instructions.

\section{Expression's isolation and quantitative RT-PCR (Q-PCR)}

For mRNA expression, total RNA was extracted from IMCD3 cells using TRIzol reagent (Invitrogen, USA). Then, cDNA was synthesized from mRNA using the iScript cDNA synthesis kit (Bio-Rad, USA). The qRTPCR assays were performed using SYBR PrimeScript RT-PCR Kit (Takara Bio, China). The sequences of the PCR primers are shown as follows: DJ-1 forward primer, 5'-TGCTGAAACTCTGCCATGTGAACC-3'; reverse primer, 5'-CCTGCTTGCCGAATATCAT-3'. GAPDH (endogenous control) forward primer, 5'-TCCTCTGACTTCAACAGCGACAC-3'; reverse primer, 5'-CACCCTGTTGCTGTAGCCAAATTC-3'.

\section{Protein extraction and Western blot analysis}

Whole-cell protein was extracted from kidneys or IMCD3 cells using Radio immunoprecipitation assay (RIPA) buffer (Thermo Scientific, USA). An equal amount of proteins $(20 \mu \mathrm{g})$ was loaded on a sodium dodecyl sulfate polyacrylamide gel electrophoresis (SDS-PAGE) and transferred to a polyvinylidene fluoride (PVDF) membrane (Millipore, USA). The membrane was then blocked with 5\% skim milk and incubated overnight at $4{ }^{\circ} \mathrm{C}$ with anti-DJ-1 
(1:1,000, 2134S, CST, USA), anti-Ki67 (1:1,000, ab16667, Abcam, USA), anti-PCNA (1:2,000, ab18197, Abcam, USA), anti-Bax (1:500, 2772S, CST, USA), anti-Bcl-2 (1:500, ab196495, Abcam, USA), anti- $\alpha$-Smooth muscle actin (SMA, 1:1,000, ab5694, Abcam, USA), anti-fibronectin (FN1, 1:1,000, ab2413, Abcam, USA), anti-TGF- $\beta 1$ (1:1,000, ab92486, Abcam, USA), anti-p53 (1:5,000, ab26, Abcam, USA), anti-Cytc (1:5,000, ab133504, Abcam, USA), anti-cleaved-caspase 3 (1:1,000, ab2302, Abcam, USA), and anti-cleaved-caspase 9 (1:1,000, ab2324, Abcam, USA) antibodies. Subsequently, the goat-anti-mouse Horseradish Peroxidase (HRP)-conjugated IgG antibody (1:5,000, Cat. No. ab205718, Abcam, USA) was used as a secondary antibody. Finally, bands were developed with an Enhanced Chemiluminescence Kit (Thermo Scientific, USA) and quantified by Quantity One imaging software (BioRad, USA) normalized to $\beta$-actin (internal control).

\section{High-resolution respirometry}

Respiration in IMCD3 cells and mitochondria was checked with high-resolution respirometry (Oxygraph-2k, Oroboros Instruments, Innsbruck, Austria) equipped with two chambers using a chamber volume set to $2 \mathrm{~mL}$. The protocol was broadly following the previously published study (8). Briefly, IMCD3 cells were re-suspended in the MiR05 solution and transferred separately to oxygraph chambers at a density of $1 \times 10^{6}$ cells $/ \mathrm{mL}$. Firstly, routine respiration ( $\mathrm{R}$-value) was assessed. After respiration stabilized, the plasma membrane was permeabilized with digitonin, followed by the serial addition of glutamate, malate, and ADP to measure the complex I (CI)-dependent oxidative phosphorylation (OXPHOS). Then, succinate (S) was added to induce maximal OXPHOS capacity (CI + II OXPHOS). CI + II-linked electron transfer system (ETS) capacity was measured by uncoupling with carbonyl cyanide p-trifluoromethoxyphenylhydrazone (FCCP). Rotenone was used to inhibit the Complex I and then measured the complex II (CII)-linked ETS. Residual oxygen consumption rate was determined by the inhibition of Complex III with antimycin A. Complex IV (CIV)-linked ETS capacity was measured by adding the CIV substrates ascorbate sodium salt and N, N, N',N'-tetramethyl-p-phenylenediamine dihydrochloride (TMPD).

\section{Mitochondrial $\mathrm{Ca}^{2+}$ uptake assay}

Mitochondrial $\mathrm{Ca}^{2+}$ uptake capacity was measured by using the protocol of mitochondrial $\mathrm{Ca}^{2+}$ uptake assay, following the previously published study (8). Briefly, cells were digested and re-suspended using the extracellular solution. Then, cell lines were treated with $5 \mu$ M Rhod-2 AM and $0.025 \% \mathrm{~F}-127$ for $30 \mathrm{~min}$ in the dark and washed with the extracellular solution. Subsequently, digitonin (0.02\%) was added to permeabilize cells, followed by the serial addition of agonist (ATP) and $\mathrm{Ca}^{2+}$ for the next treatment. Fluorescence was measured at an emission wavelength of $581 \mathrm{~nm}$ and an excitation wavelength of $552 \mathrm{~nm}$.

\section{Measurement of Mitochondrial complex I activity}

The Mitochondrial Complex I Activity Assay Kit determined the mitochondrial complex I activity of the IMCD3 cells (Merck Millipore, Darmstadt, Germany) according to the manufacturer's protocol. Mitochondrial complex I activity was detected at $450 \mathrm{~nm}$ absorbance using a microplate reader (Bio-Rad, USA).

\section{Statistical analysis}

GraphPad Prism software version 7.0 (GraphPad Software, La Jolla, CA) was used to perform the statistical analysis. Results were shown as mean \pm mean of standard error (SEM) from three independent experiments. Statistical analysis was performed using Student's $t$-test for pairwise comparisons or analysis of variance (ANOVA) followed by Tukey's post hoc test for multiple comparisons. Differences were considered statistically significant when $\mathrm{P}<0.05$.

\section{Results}

\section{Df-1 knock-down enhanced the proliferation of IMCD3 cells}

To investigate the role of $D \mathcal{F}-1$ in ADPKD, we firstly detected its function in vitro. Df-1 was knocked down in IMCD3 cells, and then the decreased expression of DJ-1 was confirmed by qRT-PCR and western blot, respectively (Figure 1A,B). After Df-1 knock-down, cell proliferation and the expression of proliferation-related proteins were measured to evaluate the effects of $D f-1$ on cell proliferation. We observed that the knock-down of $D \mathcal{f}-1$ significantly increased the proliferation rate of IMCD3 cells compared to the wild-type cells $(\mathrm{P}<0.05$, Figure $1 C)$. Meanwhile, the knock-down of $D f-1$ promoted the protein expression of Ki67 and PCNA $(\mathrm{P}<0.001$, Figure 1D). The results concluded that the knock-down of $D \mathcal{F}-1$ enhanced IMCD3 cell proliferation, showing that DJ-1 may participate in the progression of ADPKD. 
A
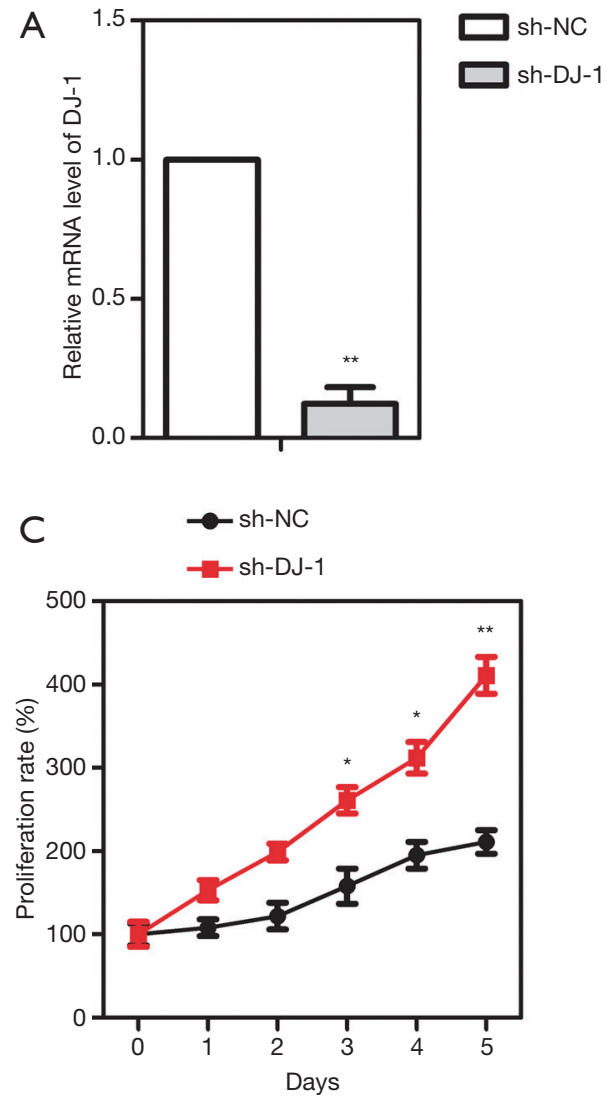

B

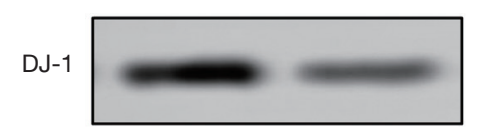

$\beta$-actin

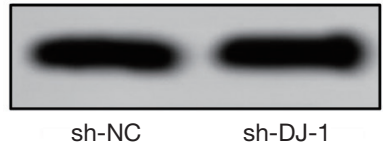

sh-NC

sh-DJ-1
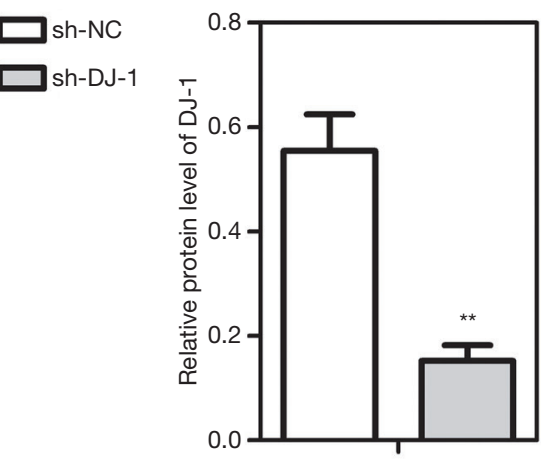

$\mathrm{D}$
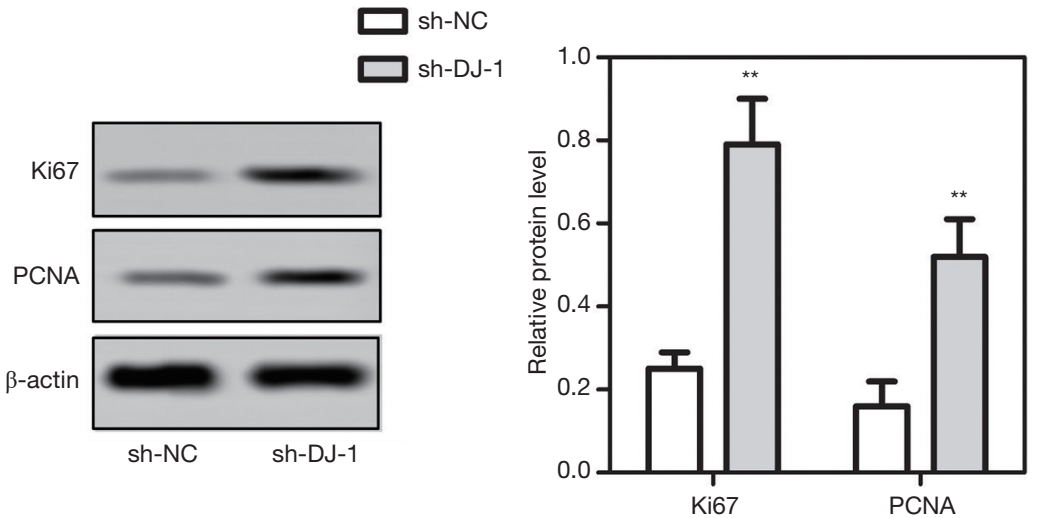

Figure 1 DJ-1 knock-down enhanced the proliferation of IMCD3 cells. (A) mRNA levels of DJ-1 were detected by qRT-PCR analysis in IMCD3 cells after shRNA transfection; (B) protein expression levels of DJ-1 were detected by Western blot in IMCD3 cells after shRNA transfection; (C) after transfection, the cell proliferation rate of IMCD3 cells was determined by CCK-8 assay; (D) after transfection, the expression of proliferation-related proteins (Ki67 and PCNA) was measured by western blot using $\beta$-actin as an internal control. Data were expressed in mean \pm SEM of three separate experiments. *, $\mathrm{P}<0.05,{ }^{* *}, \mathrm{P}<0.01$, compared to DJ-1-WT (wild type).

\section{Df-1 knock-down induced the cell apoptosis, mitochondrial dysfunction and oxidative stress of IMCD 3 cells}

To further assess the function of $D \mathcal{F}-1$ in cell phenotype and mitochondrial metabolism in vitro, the cell apoptosis, and the expression of apoptosis-related proteins was measured after cell transfection. As shown in Figure 2A, compared with the wild-type cells, the apoptosis of transfected IMCD 3 cells with shRNA-Df-1 significantly enhanced. Accordingly, the knock-down of Df-1 markedly increased the expression levels of Bax, intracellular Cyt c, cleavedcaspase 3, and cleaved-caspase 9, but suppressed the Bcl-2 expression (Figure 2B). Furthermore, the mitochondrial metabolism was evaluated using the high-resolution respirometry, $\mathrm{Ca}^{2+}$ absorption in mitochondria, and the activity of the respiratory enzyme. The results showed that the knock-down of $D f-1$ suppressed the ROUTINE respiration, the CI-linked OXPHOS capacity, the CI+IIlinked OXPHOS capacity, the CI + II-linked ETS capacity, and the CII-linked ETS capacity, while had no effect on the LEAK respiration, CII-linked OXPHOS capacity and the CIV-linked ETS capacity (Figure 2C). Moreover, we found that the inhibition of DJ-1 both significantly inhibited the $\mathrm{Ca}^{2+}$ uptake ability in mitochondria (Figure $2 D$ ) and mitochondrial complex I activity (Figure 2E). In addition, the inhibition of DJ-1 obviously suppressed the levels of SOD (Figure 2F), but enhanced the MDA levels (Figure 2G), implicating the activation of oxidative stress. The results concluded that the knock-down of DF-1 induced the cell apoptosis, mitochondrial dysfunction and oxidative stress of IMCD3 cells. 
A

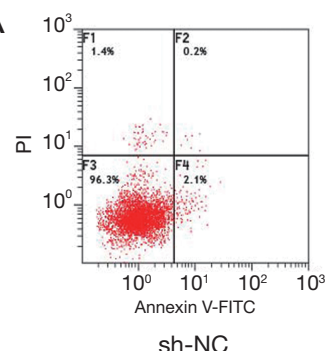

B

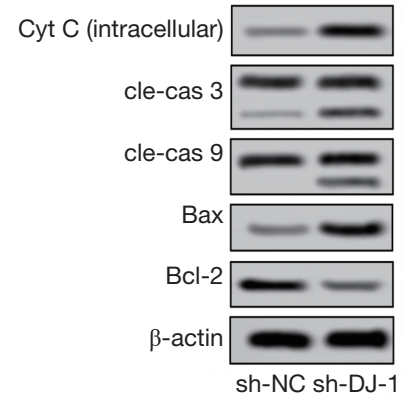

C

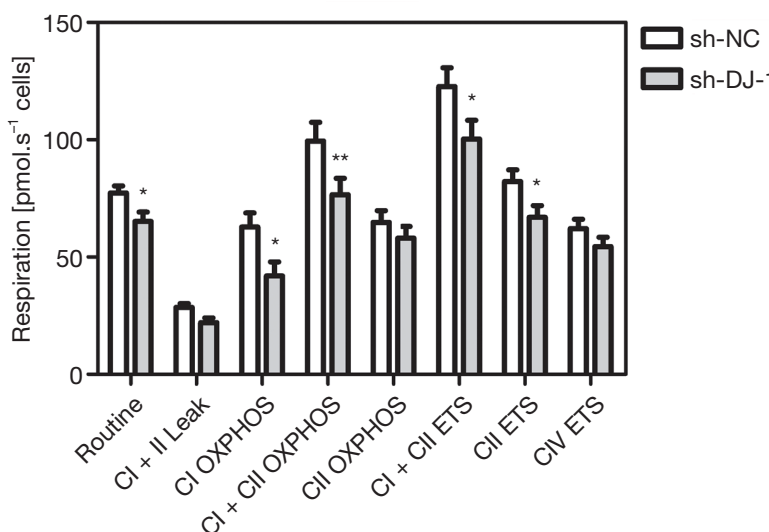

$E$

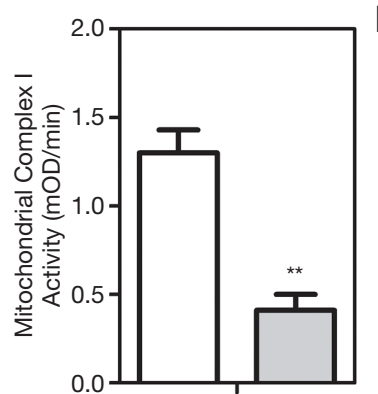

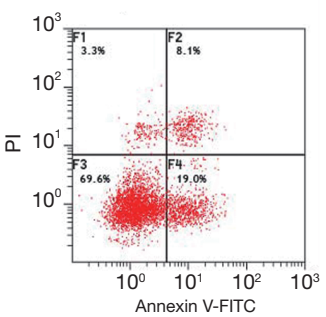

sh-DJ-1

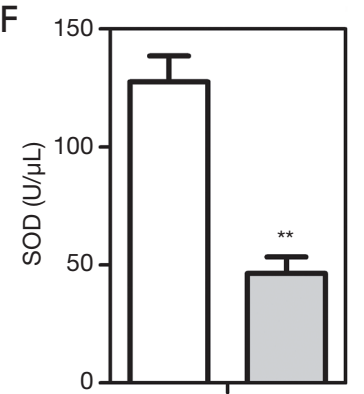

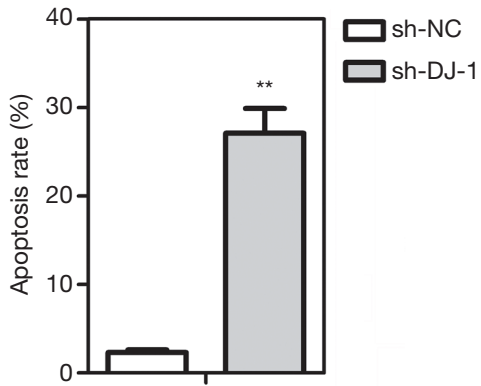
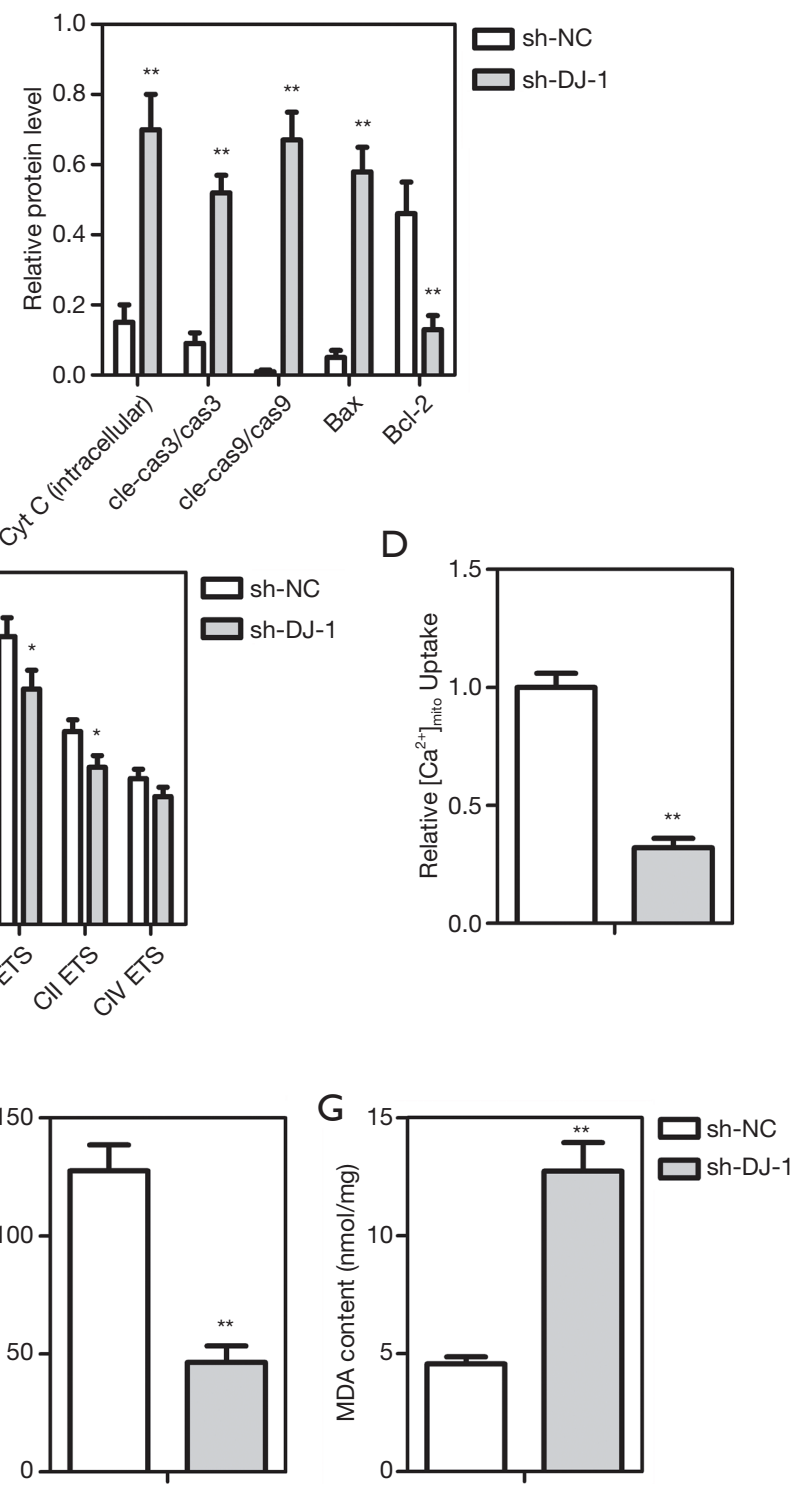

Figure 2 DJ-1 knock-down induced the cell apoptosis, mitochondrial dysfunction of IMCD3 cells. (A) After transfection, the cell apoptosis was detected by flow cytometry assay. (B) after transfection, the expression of apoptosis-related proteins was measured by western blot using $\beta$-actin as an internal control; (C) the effects of DJ-1 knock-down on mitochondrial respiratory function in IMCD3 cells were assessed by high-resolution respirometry; (D) the effects of DJ-1 knock-down on $\mathrm{Ca}^{2+}$ absorption in mitochondria; (E) the effects of DJ-1 knock-down on mitochondrial complex I activity; (F) the effects of DJ-1 knock-down on SOD levels; (G) the effects of DJ-1 knock-down on MDA levels. Data were expressed in mean \pm SEM of three separate experiments. *, $\mathrm{P}<0.05$, **, $\mathrm{P}<0.01$, compared to DJ-1-WT (wild type). 
A

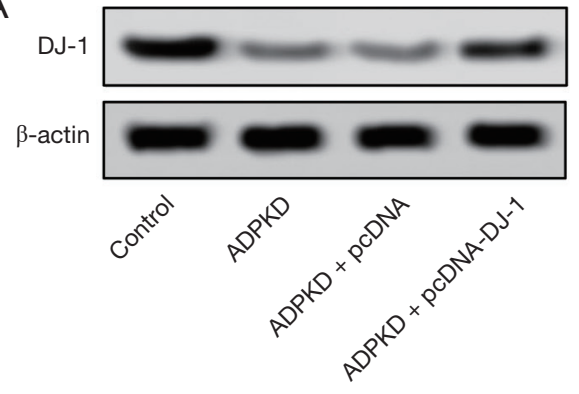

B

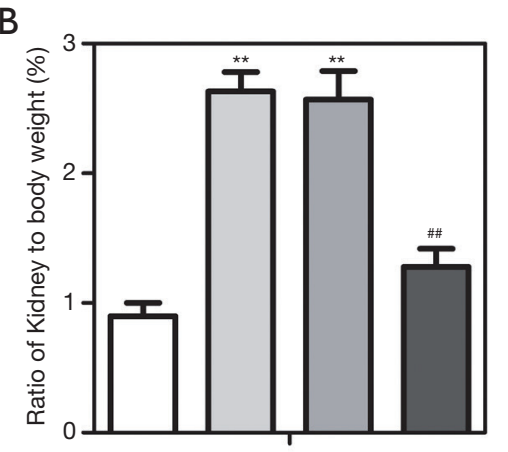

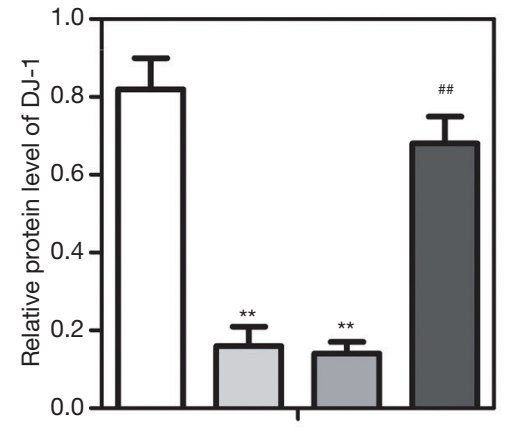

C

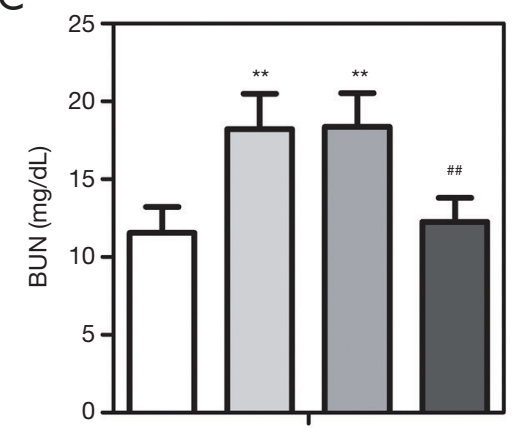

$\mathrm{D}$

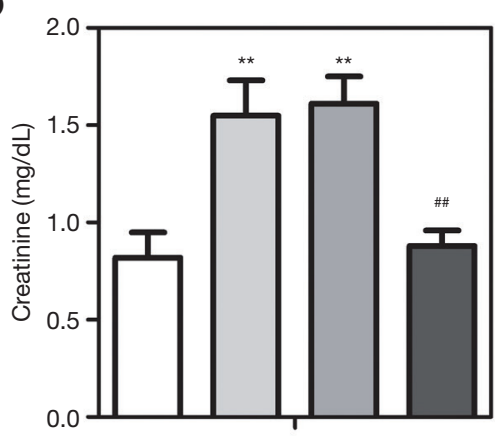

$E$

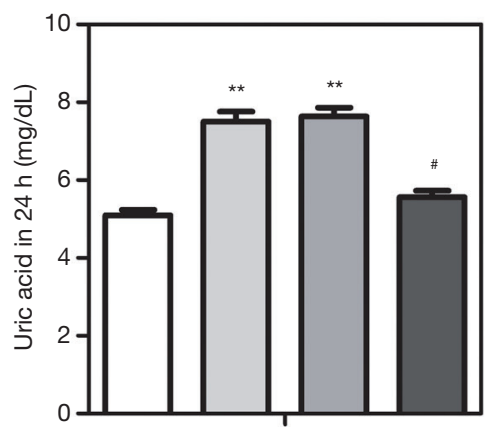

$\mathrm{F}$

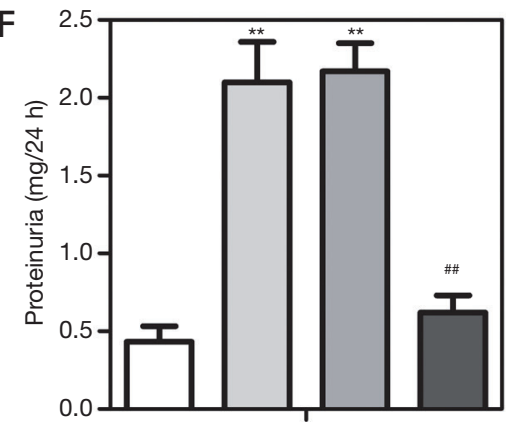

Figure 3 Overexpression of DJ-1 attenuated the renal dysfunction ADPKD models. To understand the function of DJ-1 in the ADPKD progression, DJ-1 was overexpression in ADPKD models. Kidney extracts were obtained for analysis. (A) The expression of DJ-1 was measured by western blot. (B) Kidney-weight-to-body-weight ratios, (C) serum BUN levels, (D) serum creatinine levels, (E) serum uric acid levels, and (F) serum proteinuria levels were shown to evaluate the renal function of ADPKD models. Data were expressed in mean \pm SEM ( $\mathrm{n}=10$ per group). ${ }^{* *}, \mathrm{P}<0.01$, compared to control; ${ }^{\#}, \mathrm{P}<0.05,{ }^{\# \#}, \mathrm{P}<0.01$, compared to ADPKD + pcDNA.

\section{Overexpression of DF-1 attenuated the renal dysfunction in $A D P K D^{p c D N A-D F-1}$ mouse}

The wild-type ADPKD mice models and transgenic ADPKD models with overexpressing DJ-1 were constructed to simulate in vivo conditions to supply in vivo evidence for the function of $D \mathcal{F}-1$ in the ADPKD progression. Firstly, western blot assay for kidney tissue proved DJ-1 was down-regulated in ADPKD models, and meanwhile, DJ-1 was successfully up-regulated in $\mathrm{ADPKD}^{\mathrm{pcDNA}-\mathrm{DJ}-1}$ mouse
(Figure 3A). Then, we observed the kidney-weight-tobody-weight ratios, serum BUN, creatinine, uric acid, and proteinuria levels were all enhanced in ADPKD models compared with blank control, which was attenuated by the overexpression of DJ-1 in ADPKD ${ }^{\text {pcDNA-DJ-1 }}$ mouse (Figure 3B,C,D,E,F). The results concluded that $D \mathcal{F}-1$ participated in the pathogenesis of ADPKD in vivo, and its overexpression could attenuate the renal dysfunction in ADPKD models. 


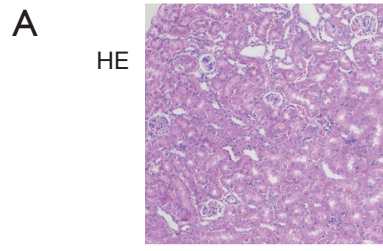

Control

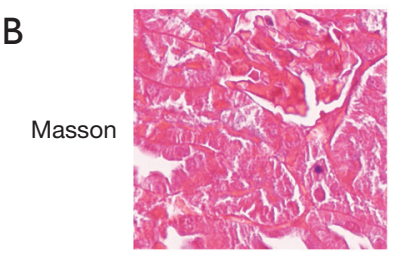

Control

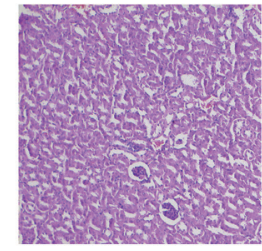

ADPKD

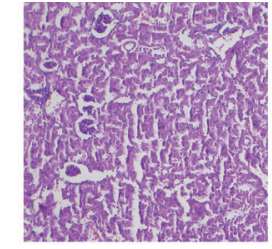

ADPKD + pcDNA

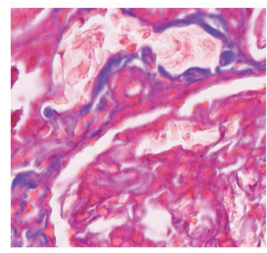

ADPKD

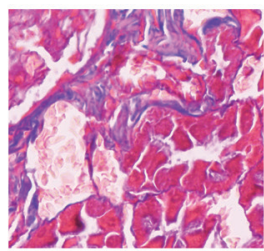

ADPKD + pcDNA

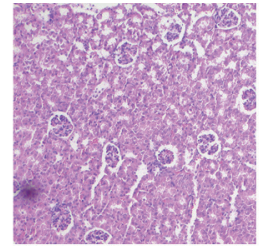

ADPKD + pcDNA-DJ-1

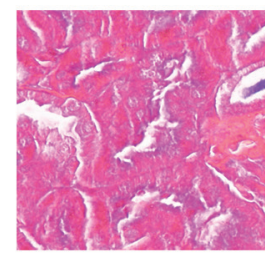

ADPKD + pcDNA-DJ-1

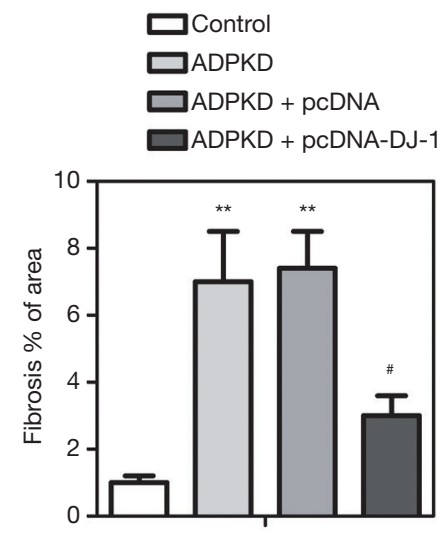

C
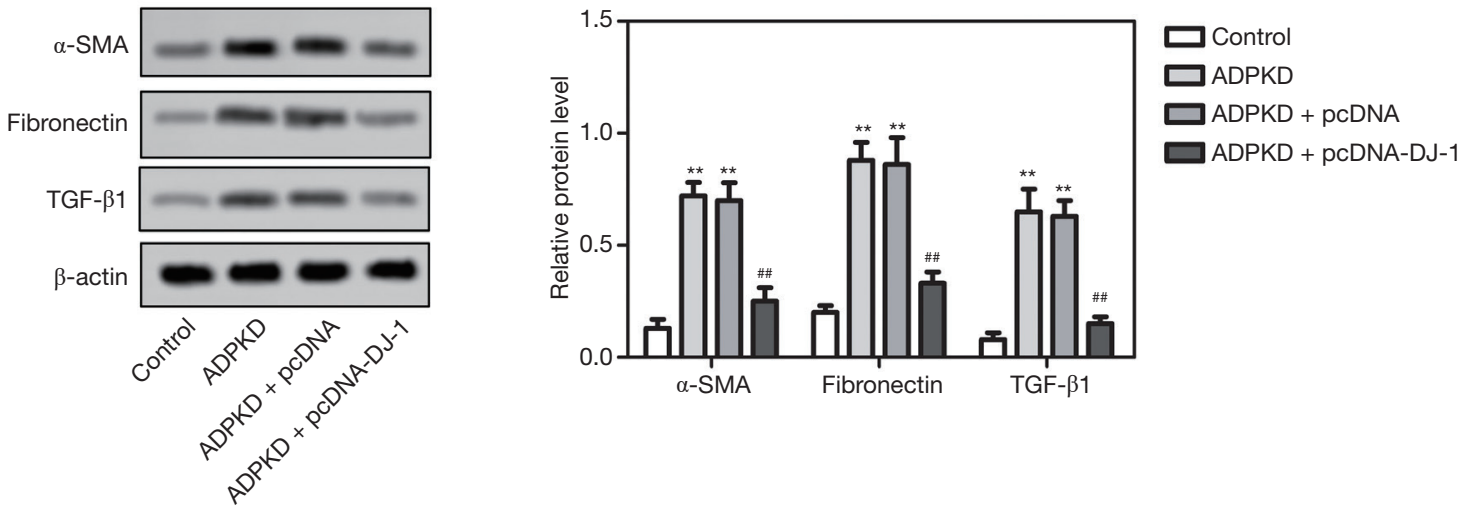

Figure 4 Overexpression of DJ-1 attenuated the pathological damage to kidney tissue in vivo. (A) H\&E staining (magnification: $\times 200$ ) and (B) Masson staining (magnification: $\times 400$ ) of kidney sections from the 28-day-old wild-type and transgenic ADPKD models. (C) The expression levels of fibrosis-related proteins in wild-type and transgenic ADPKD models were measured by western blot. Data were expressed in mean \pm SEM ( $\mathrm{n}=10$ per group). ** $\mathrm{P}<0.01$, compared to control. ${ }^{*}, \mathrm{P}<0.05$, ${ }^{\text {\# }}, \mathrm{P}<0.01$, compared to ADPKD + pcDNA.

\section{Overexpression of DF-1 attenuated the pathological damage to kidney tissue in ADPKD $D^{p c D N A-D f-1}$ mouse}

Subsequently, to evaluate whether Df-1 affects the cyst growth and renal tissue fibrosis in ADPKD models, $\mathrm{H} \& \mathrm{E}$ staining, Masson staining, and the expression of fibrosisrelated proteins were detected in the treated and un-treated ADPKD models. We observed cyst growth and renal fibrosis in ADPKD mice compared with the control group (Figure $4 A, B$ ), accompanied by the up-regulation of fibrosis markers, including $\alpha$-SMA, FN1, and TGF- $\beta 1$ (Figure $4 C$ ). Notably, the overexpression of DJ-1 attenuated cyst growth and reduced renal fibrosis rate, compared with the negative control (Figure 4A,B). Besides, the overexpression of DJ-1 reversed the ADPKD-induced up-regulation of $\alpha$-SMA, FN1, and TGF- $\beta 1$ (Figure $4 C$ ). The results concluded that DJ-1 overexpression could attenuate the cyst growth and renal tissue fibrosis in ADPKD models.

\section{Overexpression of D7-1 inbibited the excessive proliferation and apoptosis of BCECs by $p 53$ signaling pathway in ADPKD ${ }^{p c D N A-D f-1}$ mouse}

Kidney extracts from mice models were used for the later detection to gain insights into whether Df-1 alleviates the renal function and pathological process of ADPKD by regulating cell proliferation and apoptosis in vivo. Firstly, the western blot assay showed the expression levels of Ki67 and PCNA were up-regulated in ADPKD models compared with blank control (Figure $5 A$ ). Whereas, the expression levels of Ki67 and PCNA in transgenic $\mathrm{ADPKD}^{\mathrm{pcDNA}-\mathrm{DJ}-1}$ models with overexpressing DJ-1 were significantly inhibited compared with negative control 
A
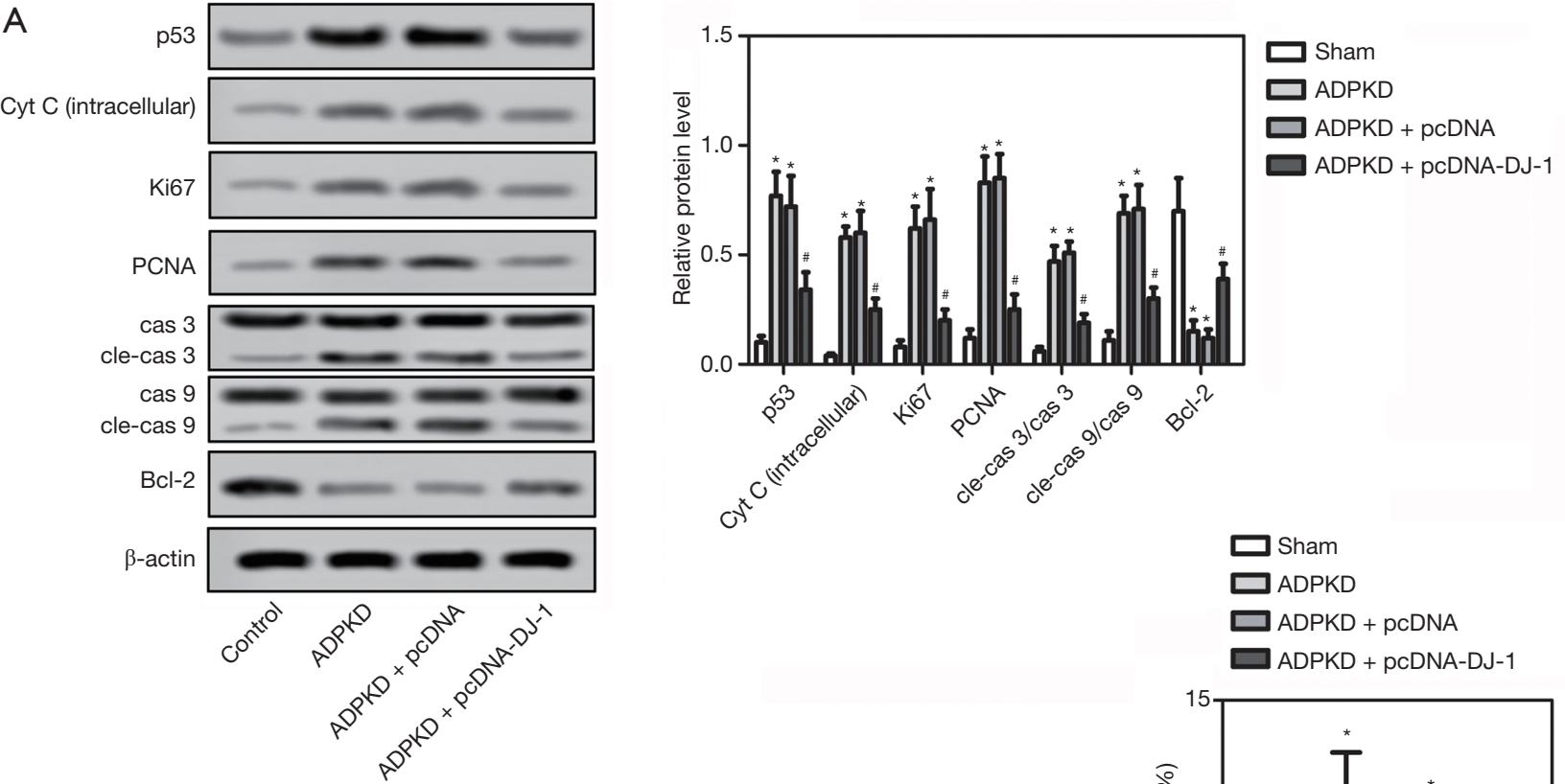

B
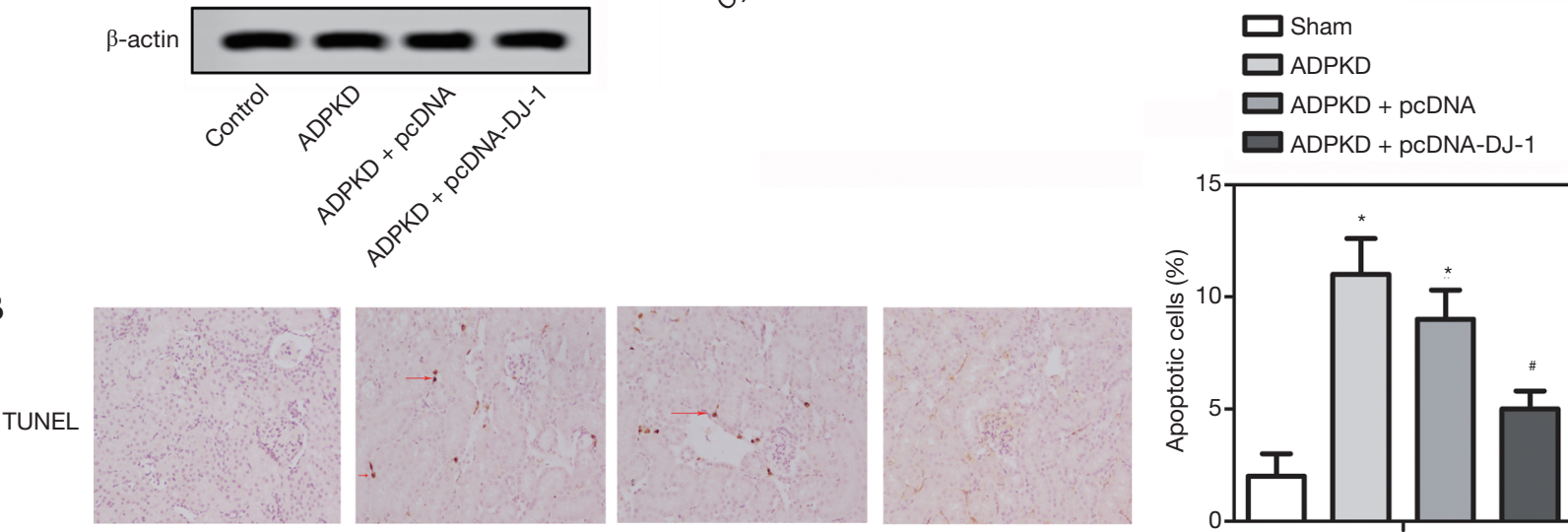

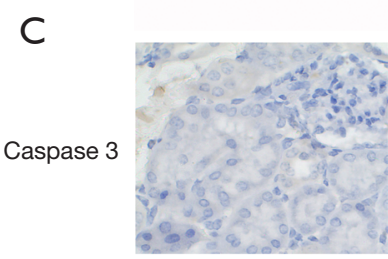

Control

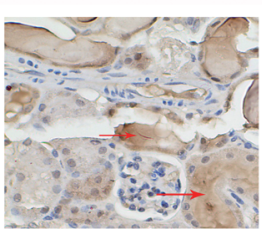

ADPKD

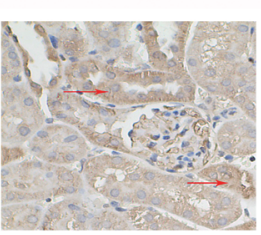

ADPKD + pcDNA
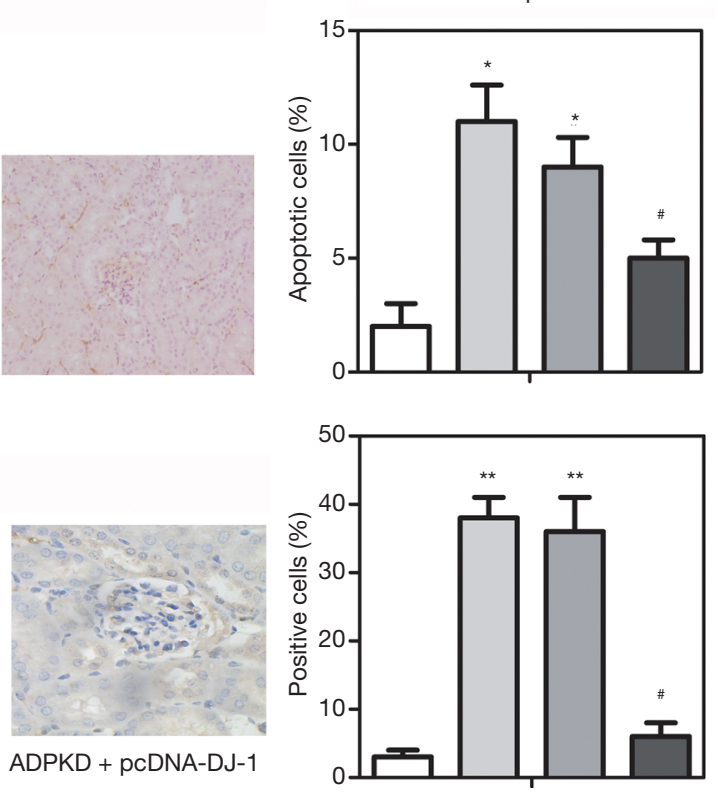

Figure 5 Overexpression of DJ-1 inhibited the excessive proliferation and apoptosis of renal epithelial cells from ADPKD models in vivo. Kidney extracts were obtained to evaluate the effects of DJ-1 on proliferation and apoptosis in vivo. (A) The expression of proliferation- and apoptosis-related proteins were measured by western blot using $\beta$-actin as an internal control. (B) Cell apoptosis rate detected by TUNEL staining. Red arrows indicate TUNEL-positive cells (apoptotic cells). Magnification: $\times 100$. (C) Immunohistochemical staining determined the expression of cleaved caspase-3. Red arrows indicate cleaved caspase 3-positive cells. Magnification: $\times 400$. Data were expressed in mean $\pm \mathrm{SEM}$ ( $\mathrm{n}=10$ per group). *, $\mathrm{P}<0.05,{ }^{* *}, \mathrm{P}<0.01$, compared to control; ${ }^{*}, \mathrm{P}<0.05$, compared to ADPKD + pcDNA.

(Figure 5A). Similarly, the cell apoptosis rate was enhanced in ADPKD models, but both have been markedly reversed by the overexpression of DJ-1 (Figure 5B). Furthermore, we preliminary explored the downstream regulatory pathway that DJ-1 may be involved, due to DJ-1 mediating the apoptosis in ADPKD. Thus, the proteins in the p53 signaling pathway were detected by western blot.
As expected, we observed that DJ-1 down-regulation in ADPKD models was accompanied by the up-regulation of p53 and Bcl-2, as well as the down-regulation of intracellular Cyt c, cleaved-caspase 3 and cleaved caspase 9 (Figure $5 A$ ). However, this trend of expression change was reversed by DJ-1 overexpression in ADPKD ${ }^{\text {pcDNA-DJ-1 }}$ mice (Figure 5A). Meanwhile, the IHC staining showed 
comparable results with western blot assay (Figure 5C). The results concluded that DJ-1 overexpression inhibited excessive proliferation and apoptosis of BCECs by the p53 signaling pathway in ADPKD models.

\section{Discussion}

At present, the pathogenesis of ADPKD is still not to be fully elucidated despite many studies. Mitochondrial dysfunction has emerged as a characteristic of ADPKD in the past decade (6). It is widely recognized that $D F-1$ is crucial for mitochondrial function (17). Despite the identification of many dysregulated pathways in ADPKD (23), the physiological functions of DJ-1 on the mitochondria metabolism in ADPKD remains elusive. Herein, we investigated the DF-1 role in ADPKD pathogenesis both in vitro and in vivo for the first time. Our study preliminary proved that overexpression of DJ-1 attenuates disease progression by the $\mathrm{p} 53$ signaling pathway in ADPKD mouse models, which has the potential to be a novel drug target for ADPKD.

The most striking finding of the present study was that DJ-1 was down-regulated in ADPKD models and regulated the progression of ADPKD. DJ-1, as a cytoprotective protein, is widely distributed in various organs, including the brain, kidney, spleen, and heart (24). Generally, DJ-1 was highly expressed in reactive astrocytes of patients with neurodegenerative diseases (25), as well as in various malignant cells, including HeLa cells and lung cancer cells (25-27). The abnormal expression of DJ-1 in these diseases was inconsistent with our result, which may occur due to the different pathological mechanisms of different diseases. Indeed, there was an article about the down-regulation of DJ-1 expression in tumors (28). To date, it is worth noting there remains a paucity of information regarding the role of Df-1 in the kidney, especially in $\operatorname{ADPKD}(29,30)$. Therefore, our finding supplied a basis for the underlying role of $D \mathcal{F}-1$ in ADPKD.

More importantly, our study suggested DJ-1 overexpression attenuated renal dysfunction, cyst growth, and renal fibrosis in ADPKD mice, indicating that DJ-1 has a protective effect on ADPKD. Meanwhile, DJ-1 overexpression reversed the ADPKD-induced upregulation of fibrosis-related proteins, including $\alpha$-SMA, FN1, and TGF- $\beta 1$. Similarly, Eltoweissy et al. previously reported that the expression of DJ-1 is associated with the progression of renal fibrosis (31). However, this study did not focus on the relationship between Df-1 and ADPKD.
To our knowledge, this is the first report illuminated the protective effect of DJ-1 in ADPKD, which not only provided more information on the mechanisms of $D F-1$ but also provided novels insights into the development of ADPKD targeted therapy. Traditionally, Df-1 protects against cell death in hypoxia/reoxygenation injury (32) and Parkinson's disease (33). According to many previous studies, the protective effect of DJ-1 is attributed to its anti-oxidative stress function (24). Many pieces of evidence have demonstrated DJ-1 deficiency results in the elevation of reactive oxygen species (ROS) (34) and thereby sensitizes renal cells to oxidative stress, endoplasmic reticulum stress, and enhances cytotoxicity (35).

Interestingly, recent studies showed that oxidative stress is evident early in $\mathrm{ADPKD}$, even with preserved kidney function $(36,37)$. After the inhibition of DJ-1, we noticed that the SOD levels were decreased while MDA levels were increased, implicating that DJ-1 down-regulation could enhance the oxidative stress, which was identified with findings in other diseases $(35,38)$. Moreover, increased oxidative stress plays a functional role in cyst formation (9). On the above evidence, we considered that DJ-1 overexpression could attenuate renal dysfunction, cyst growth, and renal fibrosis may through inhibiting ROS production and decreasing oxidative stress. However, this mechanism is only on hypothesis and is still to be verified in practice.

Presently, the precise regulatory mechanism of $D \mathcal{F}-1$ in ADPKD is still entirely ambiguous. Here, we preliminarily investigated the mechanism of $D \mathcal{F}-1$ in ADPKD. An imbalance between cell proliferation and apoptosis of BCECs is considered a significant factor leading to cyst growth and renal tissue remodeling in ADPKD $(39,40)$. Therefore, we detected the effects of $D \mathcal{F}-1$ on cell proliferation and apoptosis in cell lines and ADPKD mice. As expected, we found $D \mathcal{F}-1$ deficiency induced the cell proliferation and apoptosis of IMCD3 cells accompanied by the increase of proliferation-related proteins (Ki67 and PCNA) and pro-apoptotic protein Bax, and the decrease of anti-apoptotic protein Bcl-2.

On the contrary, overexpression of DJ-1 inhibited the excessive proliferation and apoptosis of BCECs in ADPKD mice accompanied by the down-regulation of intracellular Cyt c, cleaved-caspase 3 and cleaved caspase 9. Inhibition of apoptosis has been shown to delay renal cyst growth in PKD models (41). Consistent with our study, a previous study has revealed DJ-1, as a potent nutritional antioxidant, could protect renal cells from apoptosis (31). Coincidentally, DJ-1 protects neurons from oxidative-stress-induced 
apoptosis (42). Besides, in pulmonary arterial hypertension, characterized by excessive proliferation and apoptosis resistance of pulmonary artery smooth muscle cells (PASMCs), DJ-1 overexpression reversed hypoxia-induced elevation of PASMC cell proliferation, thus alleviating hypoxia-induced PASMCs injury (43). This finding further supported our speculation that $D \mathcal{F}-1$ could attenuate the renal dysfunction and pathological damage to ADPKD models by inhibiting excessive proliferation and apoptosis.

In recent years, growing evidence suggests the mitochondrial biogenesis and their function regulation are the proximate cause of these metabolic disruptions in ADPKD to a certain extent (23). Mitochondrial dysfunction in ADPKD may result in reduced mitochondrial respiration, OXPHOS capacity, and increased production of ROS, which in turn increases cell proliferation $(23,44)$. Thereby, we hypothesized that the improvement of mitochondrial dysfunction in ADPKD might be pivotal to the ADPKD progression. Df-mediated mitochondrial dysfunction has been elaborated in Parkinson's disease $(18,19,45)$. Consistent with these previous publications, our investigation verified the induction of mitochondrial dysfunction by $D F-1$ deficiency, including respiratory depression and the decline of OXPHOS capacity, ETS capacity, $\mathrm{Ca}^{2+}$ uptake capacity, and mitochondrial complex I activity. Previously, Ishimoto et al. have demonstrated that mitochondrial abnormalities facilitate cyst formation in ADPKD (9). Accordingly, the current histopathological examination showed DJ-1 overexpression attenuates cyst growth.

Additionally, $\mathrm{Ca}^{2+}$ uptake and $\mathrm{Ca}^{2+}$ signaling is also associated with the pathogenesis of ADPKD. It is demonstrated that disrupted intracellular $\mathrm{Ca}^{2+}$ homeostasis leads to reduced intracellular $\mathrm{Ca}^{2+}$ stores, further disrupting multiple signaling pathways and leading to the cystic phenotype (46). The DJ-1 protein has a role in the oxidative stress response; under oxidative conditions, DJ-1 partially translocates to mitochondria (47). The DJ-1 mitochondrial translocation could further induce the abnormal activation of autophagy, and eventually lead to mitochondrial dysfunction (48). Thus, we here evaluated the effects of DJ-1 on mitochondrial function and found $D \mathcal{F}-1$ deficiency inhibited the mitochondrial $\mathrm{Ca}^{2+}$ uptake, which in turn regulate the cyst growth. These results showed that DJ-1 might directly regulate the mitochondrial OXPHOS capacity, ETS capacity, $\mathrm{Ca}^{2+}$ uptake, and respiratory, thus indirectly affect the mitochondrial function. Also, it is reported that the level of $\mathrm{Ca}^{2+}$ in the mitochondrial matrix regulated the ATP production (49). Subsequently, the improved effects of DJ-1 on mitochondrial dysfunction finally contribute to inhibition of excessive cell proliferation. Given the multifaceted findings in our study, we could conclude that DJ-1 acts as a protective factor by regulating mitochondrial function. Our pre-clinical study supplied a strong rationale for the development of a DJ-1 based therapeutic strategy for ADPKD. Nevertheless, the possible mechanism of $D \mathcal{F}-1$ in mitochondrial metabolism in ADPKD warrants further investigations.

Previous researchers have identified p53 as a multifunctional transcription factor that influences multiple, highly diverse cellular processes (50). Here, we tentatively assessed the effects of DJ-1 on the expression of p53 and its related proteins. As expected, the up-regulation of DJ-1 in ADPKD mice inhibited the expression of p53. Further, there was a simultaneous down-regulation of intracellular Cyt c, cleaved-caspase 3, cleaved caspase 9, and the upregulation of $\mathrm{Bcl}-2$. These results further support the inhibition of apoptosis by DJ-1.

Similarly, DJ-1 was previously proved to exert its cytoprotection through inhibiting the p53-Bax-caspase pathway $(51,52)$. The 553 promotes apoptosis by repressing the survival genes through interference with the activity of specific transcription factors or by regulating mitochondrial translocation of Bax (53). It is well-recognized that p53 directly activates the pro-apoptotic $\mathrm{Bcl}-2$ protein $\mathrm{Bax}$ and indirectly regulates the release of Cyt-c (54). Cyt-c in the cytoplasm binds to Apaf- 1 and then facilitates activation of caspase- 9 and caspase- 3 , allowing for mitochondrial membrane permeabilization and apoptosis (55). Also, p53 participates in the regulation of mitochondrially mediated cellular metabolism through its transcriptional activity (56). The p5 3 regulates the expression of nuclear-encoded synthesis of cytochrome-c oxidase $2\left(\mathrm{SCO}_{2}\right)$, which is crucial for the proper assembly and function of cytochrome-c oxidase (COX) enzyme complex in the electron transport chain (57). Given the fact that $\mathrm{p} 53$ regulates mitochondrial respiration and apoptosis $(53,58)$, we speculate here that DJ-1 attenuated the renal dysfunction and pathological damage to ADPKD by regulating cell proliferation, apoptosis and mitochondrial metabolism by $\mathrm{p} 53$ signaling pathway. Thus, targeting DJ-1 has the potential to become an alternative treatment for ADPKD.

Although we reported the role of $D F-1$ in ADPKD and clarified its molecular mechanism in the disease progression, this study is a preliminary trial. The underlying molecular mechanisms of how Df-1 participates in ADPKD pathogenesis have not been revealed entirely and remain to 
be investigated.

\section{Conclusions}

The study firstly proved DJ-1 was down-regulated in ADPKD models. Importantly, we illustrated that DJ-1 attenuated the renal dysfunction and pathological damage to ADPKD through a mechanism involving the regulation of mitochondrial function, which may be mediated by the p53 signaling pathway. This finding lays the groundwork relevant to the development of a DJ-1 based therapeutic strategy for ADPKD.

\section{Acknowledgments}

Funding: None.

\section{Footnote}

Reporting Checklist: The authors have completed the ARRIVE reporting checklist. Available at http://dx.doi. org/10.21037/atm-20-5761

Data Sharing Statement: Available at http://dx.doi. org/10.21037/atm-20-5761

Conflicts of Interest: All authors have completed the ICMJE uniform disclosure form (available at http://dx.doi. org/10.21037/atm-20-5761). The authors have no conflicts of interest to declare.

Ethical Statement: The authors are accountable for all aspects of the work in ensuring that questions related to the accuracy or integrity of any part of the work are appropriately investigated and resolved. All animal procedures were performed following the NIH Guide for the Care and Use of Laboratory Animals and approved by the Ethics Review Committee of Beijing Friendship Hospital, Capital Medical University approval number: SYXK[Jing]2017-0019\}.

Open Access Statement: This is an Open Access article distributed in accordance with the Creative Commons Attribution-NonCommercial-NoDerivs 4.0 International License (CC BY-NC-ND 4.0), which permits the noncommercial replication and distribution of the article with the strict proviso that no changes or edits are made and the original work is properly cited (including links to both the formal publication through the relevant DOI and the license). See: https://creativecommons.org/licenses/by-nc-nd/4.0/.

\section{References}

1. Chebib FT, Torres VE. Autosomal Dominant Polycystic Kidney Disease: Core Curriculum 2016. Am J Kidney Dis 2016;67:792-810.

2. Torres VE, Harris PC, Pirson Y. Autosomal dominant polycystic kidney disease. Lancet 2007;369:1287-301.

3. Makhlough A, Shekarchian S, Moghadasali R, et al. Safety and tolerability of autologous bone marrow mesenchymal stromal cells in ADPKD patients. Stem Cell Res Ther 2017;8:116.

4. Yuan C, Xiang L, Bai R, et al. MiR-195 restrains lung adenocarcinoma by regulating $\mathrm{CD} 4+\mathrm{T}$ cell activation via the CCDC88C/Wnt signaling pathway: a study based on the Cancer Genome Atlas (TCGA), Gene Expression Omnibus (GEO) and bioinformatic analysis. Ann Transl Med 2019;7:263.

5. Rowe I, Chiaravalli M, Mannella V, et al. Defective glucose metabolism in polycystic kidney disease identifies a new therapeutic strategy. Nat Med 2013;19:488-93.

6. Marchi S, Pinton P. The mitochondrial calcium uniporter complex: molecular components, structure and physiopathological implications. J Physiol 2014;592:829-39.

7. Magistroni R, Boletta A. Defective glycolysis and the use of 2-deoxy-D-glucose in polycystic kidney disease: from animal models to humans. J Nephrol 2017;30:511-9.

8. Li Z, Zhou J, Li Y, et al. Mitochondrial TRPC3 promotes cell proliferation by regulating the mitochondrial calcium and metabolism in renal polycystin-2 knockdown cells. Int Urol Nephrol 2019;51:1059-70.

9. Ishimoto Y, Inagi R, Yoshihara D, et al. Mitochondrial abnormality facilitates cyst formation in autosomal dominant polycystic kidney disease. Mol Cell Biol 2017;37:e00337-17.

10. Csordás G, Weaver D, Hajnoczky G. Endoplasmic Reticulum-Mitochondrial Contactology: Structure and Signaling Functions. Trends Cell Biol 2018;28:523-40.

11. Giorgi C, De Stefani D, Bononi A, et al. Structural and functional link between the mitochondrial network and the endoplasmic reticulum. Int J Biochem Cell Biol 2009;41:1817-27.

12. Kaufman RJ, Malhotra JD. Calcium trafficking integrates endoplasmic reticulum function with mitochondrial bioenergetics. Biochim Biophys Acta 2014;1843:2233-9.

13. Pizzo P, Pozzan T. Mitochondria-endoplasmic reticulum 
choreography: structure and signaling dynamics. Trends Cell Biol 2007;17:511-7.

14. Kuo IY, Brill AL, Lemos FO, et al. Polycystin 2 regulates mitochondrial $\mathrm{Ca}(2+)$ signaling, bioenergetics, and dynamics through mitofusin 2. Sci Signal 2019; 12:eaat7397.

15. Hayashi T, Ishimori C, Takahashi-Niki K, et al. DJ-1 binds to mitochondrial complex I and maintains its activity. Biochem Biophys Res Commun 2009;390:667-72.

16. McCoy MK, Cookson MR. DJ-1 regulation of mitochondrial function and autophagy through oxidative stress. Autophagy 2011;7:531-2.

17. Hao LY, Giasson BI, Bonini NM. DJ-1 is critical for mitochondrial function and rescues PINK1 loss of function. Proc Natl Acad Sci U S A 2010;107:9747-52.

18. Larsen NJ, Ambrosi G, Mullett SJ, et al. DJ-1 knock-down impairs astrocyte mitochondrial function. Neuroscience 2011;196:251-64.

19. Thomas KJ, McCoy MK, Blackinton J, et al. DJ-1 acts in parallel to the PINK1/parkin pathway to control mitochondrial function and autophagy. Hum Mol Genet 2011;20:40-50.

20. Pantner Y, Shimizu Y, Polavarapu R, et al. Abstract 306: DJ-1 Preserves Mitochondrial Function in the Ischemic Heart by Reducing the Glycation of Complex I. Circulation Research 2019;125:A306-A.

21. Salazar C, Ruiz-Hincapie P, Ruiz LM. The Interplay among PINK1/PARKIN/Dj-1 Network during Mitochondrial Quality Control in Cancer Biology: Protein Interaction Analysis. Cells 2018;7:154.

22. Capasso JM, Rivard CJ, Berl T. Silencing and overexpression of the gamma-subunit of Na-K-ATPase directly affect survival of IMCD3 cells in response to hypertonic stress. Am J Physiol Renal Physiol 2006;291:F1142-7.

23. Padovano V, Podrini C, Boletta A, et al. Metabolism and mitochondria in polycystic kidney disease research and therapy. Nat Rev Nephrol 2018;14:678-87.

24. Guzman JN, Sanchez-Padilla J, Wokosin D, et al. Oxidant stress evoked by pacemaking in dopaminergic neurons is attenuated by DJ-1. Nature 2010;468:696-700.

25. Neumann M, Muller V, Gorner K, et al. Pathological properties of the Parkinson's disease-associated protein DJ-1 in alpha-synucleinopathies and tauopathies: relevance for multiple system atrophy and Pick's disease. Acta Neuropathol 2004;107:489-96.

26. Taira T, Takahashi K, Kitagawa R, et al. Molecular cloning of human and mouse DJ-1 genes and identification of Sp1- dependent activation of the human DJ-1 promoter. Gene 2001;263:285-92.

27. Kim RH, Peters M, Jang Y, et al. DJ-1, a novel regulator of the tumor suppressor PTEN. Cancer Cell 2005;7:263-73.

28. Zhang D, Lim SG, Koay ES. Proteomic identification of down-regulation of oncoprotein DJ-1 and proteasome activator subunit 1 in hepatitis B virus-infected welldifferentiated hepatocellular carcinoma. Int J Oncol 2007;31:577-84.

29. Yao Y, Wei H, Liu L, et al. Upregulated DJ-1 promotes renal tubular EMT by suppressing cytoplasmic PTEN expression and Akt activation. J Huazhong Univ Sci Technolog Med Sci 2011;31:469.

30. Sitaram RT, Cairney CJ, Grabowski P, et al. The PTEN regulator DJ-1 is associated with hTERT expression in clear cell renal cell carcinoma. Int J Cancer 2009;125:783-90.

31. Eltoweissy M, Dihazi GH, Muller GA, et al. Protein DJ-1 and its anti-oxidative stress function play an important role in renal cell mediated response to profibrotic agents. Mol Biosyst 2016;12:1842-59.

32. Shen ZY, Sun Q, Xia ZY, et al. Overexpression of DJ-1 reduces oxidative stress and attenuates hypoxia/ reoxygenation injury in NRK-52E cells exposed to high glucose. Int J Mol Med 2016;38:729-36.

33. Ariga H, Takahashi-Niki K, Kato I, et al. Neuroprotective function of DJ-1 in Parkinson's disease. Oxid Med Cell Longev 2013;2013:683920.

34. Cuevas S, Zhang Y, Yang Y, et al. Role of renal DJ-1 in the pathogenesis of hypertension associated with increased reactive oxygen species production. Hypertension 2012;59:446-52.

35. Yokota T, Sugawara K, Ito K, et al. Down regulation of DJ-1 enhances cell death by oxidative stress, ER stress, and proteasome inhibition. Biochem Biophys Res Commun 2003;312:1342-8.

36. Andries A, Daenen K, Jouret F, et al. Oxidative stress in autosomal dominant polycystic kidney disease: player and/ or early predictor for disease progression? Pediatr Nephrol 2019;34:993-1008.

37. Menon V, Rudym D, Chandra P, et al. Inflammation, oxidative stress, and insulin resistance in polycystic kidney disease. Clin J Am Soc Nephrol 2011;6:7-13.

38. Kahle PJ, Waak J, Gasser T. DJ-1 and prevention of oxidative stress in Parkinson's disease and other agerelated disorders. Free Radic Biol Med 2009;47:1354-61.

39. Lee EJ. Cell Proliferation and Apoptosis in ADPKD. In: Park JH, Ahn C, editors. Cystogenesis. Singapore: Springer Singapore, 2016:25-34. 
40. Reif GA, Wallace DP. ADPKD cell proliferation and $\mathrm{Cl}(-)$-dependent fluid secretion. Methods Cell Biol 2019;153:69-92.

41. Zhou JX, Li X. Apoptosis in Polycystic Kidney Disease: From Pathogenesis to Treatment. In: Li X, editor. Polycystic Kidney Disease. Brisbane (AU): Codon Publications; 2015.

42. Ma J, Wu R, Zhang Q, et al. DJ-1 interacts with RACK1 and protects neurons from oxidative-stress-induced apoptosis. Biochem J 2014;462:489-97.

43. Gao W, Shao R, Zhang X, et al. Up-regulation of caveolin-1 by DJ-1 attenuates rat pulmonary arterial hypertension by inhibiting TGFbeta/Smad signaling pathway. Exp Cell Res 2017;361:192-8.

44. Picard M, Shirihai OS, Gentil BJ, et al. Mitochondrial morphology transitions and functions: implications for retrograde signaling? Am J Physiol Regul Integr Comp Physiol 2013;304:R393-406.

45. Dodson MW, Guo M. Pink1, Parkin, DJ-1 and mitochondrial dysfunction in Parkinson's disease. Curr Opin Neurobiol 2007;17:331-7.

46. Harris PC, Torres VE. Genetic mechanisms and signaling pathways in autosomal dominant polycystic kidney disease. J Clin Invest 2014;124:2315-24.

47. Giorgi C, Romagnoli A, Pinton P, et al. Ca2+ signaling, mitochondria and cell death. Curr Mol Med 2008;8:119-30.

48. Huang L, Hou Y, Wang L, et al. p38 Inhibitor Protects Mitochondrial Dysfunction by Induction of DJ-1 Mitochondrial Translocation After Subarachnoid Hemorrhage. J Mol Neurosci 2018;66:163-71.

49. Boyman L, Karbowski M, Lederer WJ. Regulation of

Cite this article as: Li Z, Zhou J, Li Y, Yang F, Lian X, Liu W. Overexpression of DJ-1 alleviates autosomal dominant polycystic kidney disease by regulating cell proliferation, apoptosis, and mitochondrial metabolism in vitro and in vivo. Ann Transl Med 2020;8(18):1175. doi: 10.21037/atm-20-5761
Mitochondrial ATP Production: $\mathrm{Ca}(2+)$ Signaling and Quality Control. Trends Mol Med 2020;26:21-39.

50. Stegh AH. Targeting the p53 signaling pathway in cancer therapy - the promises, challenges and perils. Expert Opin Ther Targets 2012;16:67-83.

51. Bretaud S, Allen C, Ingham PW, et al. p53-dependent neuronal cell death in a DJ-1-deficient zebrafish model of Parkinson's disease. J Neurochem 2007;100:1626-35.

52. Fan J, Ren H, Jia N, et al. DJ-1 decreases Bax expression through repressing p53 transcriptional activity. J Biol Chem 2008;283:4022-30.

53. Nayak SK, Panesar PS, Kumar H. p53-Induced apoptosis and inhibitors of p53. Curr Med Chem 2009;16:2627-40.

54. Chipuk JE, Green DR. Cytoplasmic p53: Bax and Forward. Cell Cycle 2004;3:429-31.

55. Borutaite V, Jekabsone A, Morkuniene R, et al. Inhibition of mitochondrial permeability transition prevents mitochondrial dysfunction, cytochrome c release and apoptosis induced by heart ischemia. J Mol Cell Cardiol 2003;35:357-66.

56. Saleem A, Adhihetty PJ, Hood DA. Role of p53 in mitochondrial biogenesis and apoptosis in skeletal muscle. Physiol Genomics 2009;37:58-66.

57. Ma W, Sung HJ, Park JY, et al. A pivotal role for $\mathrm{p} 53$ : balancing aerobic respiration and glycolysis. J Bioenerg Biomembr 2007;39:243-6.

58. Matoba S, Kang JG, Patino WD, et al. p53 regulates mitochondrial respiration. Science 2006;312:1650-3.

(English Language Editor: J. Chapnick) 